\title{
Metastable random-field Ising model with exchange enhancement: A simple model for exchange bias
}

\author{
Xavier Illa, ${ }^{*}$ Eduard Vives, ${ }^{\dagger}$ and Antoni Planes ${ }^{\ddagger}$ \\ Departament d'Estructura i Constituents de la Matèria, Universitat de Barcelona Diagonal 647, Facultat de Física, 08028 Barcelona, \\ Catalonia, Spain \\ (Received 1 August 2002; published 31 December 2002)
}

\begin{abstract}
We present a simple model that allows hysteresis loops with exchange bias to be reproduced. The model is a modification of the $T=0$ random-field Ising model driven by an external field and with synchronous local relaxation dynamics. The main novelty of the model is that a certain fraction $f$ of the exchange constants between neighboring spins is enhanced to a very large value $J_{E}$. The model allows the dependence of the exchange bias and other properties of the hysteresis loops to be analyzed as a function of the parameters of the model: the fraction $f$ of enhanced bonds, the amount of the enhancement $J_{E}$, and the amount of disorder, which is controlled by the width $\sigma$ of the Gaussian distribution of the random fields.

DOI: 10.1103/PhysRevB.66.224422

PACS number(s): 75.10.Nr, 75.40.Mg, 75.50.Lk, 75.60.Ej
\end{abstract}

\section{INTRODUCTION}

Hysteresis and metastability are intriguing phenomena with implications in both fundamental and applied physics. Magnetic systems are the prototypical example of thermodynamic systems exhibiting hysteresis cycles for which different theoretical approaches have been proposed. ${ }^{1}$ Besides the classical micromagnetic analysis, based on a continuous description of the magnetic properties of the system, more recently much effort has been devoted to the study of lattice models. For example, the zero-temperature random-field Ising model (RFIM) driven by an external field with convenient metastable dynamics has been very successful in qualitatively explaining some basic properties of rate-independent hysteresis loops. ${ }^{2-5}$ The most important achievement of this model has been to give a simultaneous explanation of the effect of disorder on the hysteresis loops and the existence of Barkhaussen noise with critical properties. Less attention has been paid to the use of such models for understanding other interesting features of the hysteresis loops such as remanence, coercivity, minor loop properties, or exchange bias (EB), ${ }^{6,7}$ which is the property on which we will focus our attention here.

We present a modification of the zero-temperature RFIM that allows magnetic hysteresis loops with EB to be reproduced. The main characteristic of EB is that the hysteresis loops, represented as magnetization $m$ versus external applied field $H$, are not centered on $H=0$ but exhibit a displacement in the field axis by an amount $H_{E B}$ (exchange bias field). ${ }^{6,7}$ This property has received a lot of attention recently, since the possibility of finding systems with large EB has sparked enormous technological interest. ${ }^{8}$ Experimentally EB has been found in different magnetic systems. ${ }^{6,7}$ The basic ingredient for $\mathrm{EB}$ is the existence of interfaces between ferromagnetic (FM) and antiferromagnetic (AFM) systems, where coupling can be induced after field cooling from above the Néel temperature of the antiferromagnetism. This heat treatment freezes some of the magnetic moments at the interface which are supposed to be responsible for the occurrence of EB. The prototype is a FM/AFM bilayer, for in- stance, $\mathrm{Co} / \mathrm{CoO},{ }^{9} \mathrm{NiFe} / \mathrm{NiMn},{ }^{10} \mathrm{Fe} / \mathrm{FeF}_{2},{ }^{11}$ and $\mathrm{Fe} / \mathrm{MnF}_{2} .{ }^{12}$ This effect is also observed in granular systems formed by small particles with a ferromagnetic core covered by their native antiferromagnetic oxide. ${ }^{13}$

Different models have been proposed to understand EB. Although a basic qualitative explanation was given 40 years ago $^{14}$ a deep understanding of the phenomenon has not yet been achieved. ${ }^{15,16}$ Different features remain unclear: the role played by the AFM thickness, ${ }^{17,9}$ the formation of domain walls, ${ }^{18,19}$ whether the frozen spins belong to the FM or to the AFM layer, ${ }^{20}$ etc. Especially intriguing is the fact that EB not only occurs in uncompensated AFM layers which exhibit a net magnetization after being cooled, but also in compensated AFM layers with zero net interface magnetization. $^{21}$

The aim of the present paper is to introduce a very simple model with a mechanism for the explanation of EB in totally compensated layers. The model is based on a lattice spin system with metastable dynamics for which some of the exchange interactions show a marked enhancement. In Sec. II the Hamiltonian and the detailed mestastable dynamics are presented. In Sec. III we show the results of the numerical simulations. In Sec. IV we discuss the possible physical origin of the exchange enhancement. In Sec. V we compare with available experimental data and, finally, in Sec. VI we summarize and conclude.

\section{MODEL}

The model is intended to reproduce the properties of the ferromagnetic layer only. It considers the AFM part to be totally quenched, so that it does not contribute to the net magnetization (compensated AFM layer). Consequently, we consider the two-dimensional RFIM on the square lattice, although a generalization to bulk ferromagnets or thin layers could easily be implemented. Note, however, that the antiferromagnetism plays an indirect role, as will be discussed later.

The mathematical formulation of the model is very similar to the RFIM on a square lattice with size $N=L \times L$. On each lattice site we define a spin variable $S_{i}$ which takes values \pm 1 . The Hamiltonian, in reduced units, reads 


$$
\mathcal{H}=-\sum_{i j}^{n n} J_{i j} S_{i} S_{j}-\sum_{i}^{N} h_{i} S_{i}-H \sum_{i}^{N} S_{i} .
$$

The first sum is the ferromagnetic exchange contribution that extends over nearest-neighbor pairs $\left(J_{i j}>0\right)$. The second sum accounts for the interaction with quenched random fields $h_{i}$, which stands for the disorder present in any ferromagnetic system. This term includes the effect of impurities, vacancies, and interfacial disorder of any kind, as well as interactions with the quenched AFM layer that can be interpreted as local fields acting on the FM layer. The last term is the interaction with the external driving field $H$. The random fields $h_{i}$ are independent and distributed according to a Gaussian probability density:

$$
p\left(h_{i}\right)=\frac{1}{\sqrt{2 \pi} \sigma} e^{-h_{i}^{2} / 2 \sigma^{2}},
$$

where $\sigma$ is the standard deviation of the random fields and controls the amount of disorder in the system. The novelty of the model is in the values of the exchange constants $J_{i j}$, which are not equal for all spin pairs: we consider that $J_{i j}$ $=J$ except for a fraction $f$ of the bonds (selected at random) for which $J_{i j}=J_{E}>>J$. This fraction of bonds is supposed to contain the effect of the quenched antiferromagnetic layer. A physical reason for this local exchange enhancement will be discussed in Sec. IV. Such a bond distribution can be mathematically expressed as

$$
p\left(J_{i j}\right)=(1-f) \delta\left(J_{i j}-J\right)+f \delta\left(J_{i j}-J_{E}\right) .
$$

We have focused our study in the region of small values of $f$ $(f<0.06)$. The magnetization of the system is defined as $m$ $=\sum_{i=1}^{N} S_{i} / N$. For the analysis of the hysteresis loops we use the so-called synchronous local relaxation dynamics. This is the standard dynamics used in previous studies of the zerotemperature RFIM. ${ }^{2}$ Each spin $S_{i}$ flips according to the sign of its local field $H_{i}$ given by

$$
H_{i}=\sum_{j=1}^{4} J_{i j} S_{j}+H+h_{i},
$$

where the first sum extends over the four neighbors of $S_{i}$. We start with a value of $H$ large enough so that the stable situation is given by all the spins $S_{i}=1$. We decrease the external field $H$ until $H_{i}$ vanishes on a certain spin. The spin is then reversed keeping $H$ constant. This reversal may destabilize some of the neighboring spins which are then reversed simultaneously. This is the beginning of an avalanche. The avalanche proceeds until a new stable situation is reached with all the spins $S_{i}$ aligned with their respective local fields $H_{i}$. We can then continue decreasing the external field $H$.

Most of the calculated properties are averaged over a large number $\left(\sim 10^{3}\right)$ of different realizations of disorder. Averages are indicated by the symbol $\langle\cdot\rangle$. We will consider, without loss of generality, that $J=1$. Therefore, from now on, magnetic fields and energies are given in units of $J$.

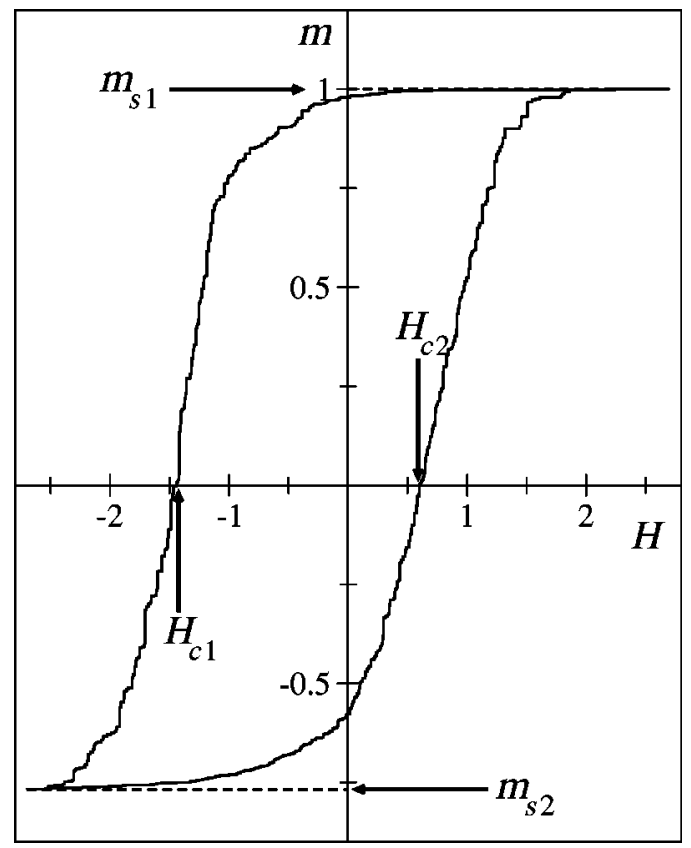

FIG. 1. Example of a hysteresis loop exhibiting exchange bias obtained from a numerical simulation of an $L=50$ system with $J_{E}$ $=20, f=0.03$, and $\sigma=1.65$. The external field has been swept from 2.7 to -2.7 . $H_{c 1}$ and $H_{c 2}$ indicate the coercive fields of the decreasing and increasing branches, respectively.

\section{RESULTS}

In Fig. 1 we present an example of a hysteresis loop obtained with the numerical simulation of a system with $L$ $=50, J_{E}=20, f=0.03$, and $\sigma=1.65$. The external field is cycled between $H= \pm 2.7$. As can be seen, the loop exhibits remarkable EB.

At first glance it may look surprising that the model defined in the previous section displays such asymmetry, since the Hamiltonian is totally symmetric under the changes $S_{i}$ $\rightarrow-S_{i}$ and $H \rightarrow-H$. The reason is that the hysteresis loop shown in Fig. 1 corresponds, strictly speaking, to a minor loop. This is revealed in Fig. 2 where the system is cycled between $H= \pm 19.4$ (which is a field that is one order-of-

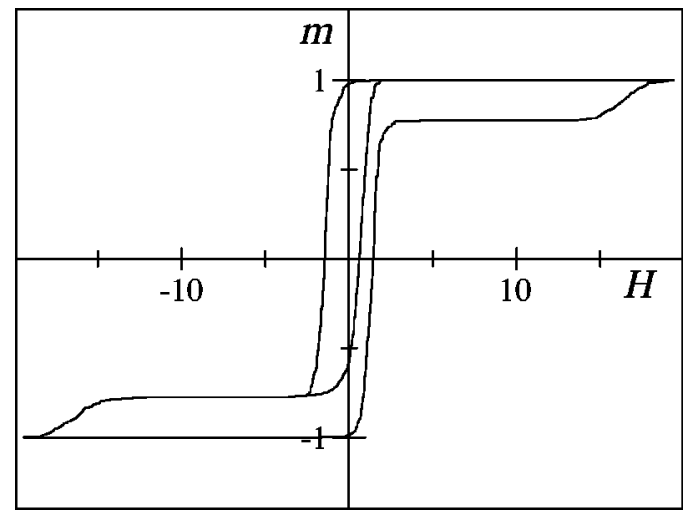

FIG. 2. The same example of Fig. 1 revealing the partial loop with exchange bias and the total loop between $H= \pm 19.4$, which is symmetric. 
magnitude larger than the coercive field $H_{c 1}$ ). Due to the existence of a tiny fraction of very large exchange interactions, the total loop exhibits long, flat plateaux in which the system behaves reversibly exactly as if it was saturated. Only when cycling between extremely large values of the external field does one obtain the symmetric hysteresis loop. Therefore, the loops with EB are incomplete loops and are accompanied by a magnetization shift.

In order to perform a quantitative analysis of this behavior, averaging over different realizations of disorder is carried out. The hysteresis loops are systematically obtained according to the following protocol: decreasing the field from $H=+\infty$ to $2 H_{c 1}<0$ and increasing the field again to $H=+\infty$, where $H_{c 1}$ is the coercive field in the decreasing branch. We also compute the pseudocoercive field $H_{c 2}$ in the increasing branch (see Fig. 1).

The criteria for choosing the value $2 H_{c 1}$ as a returning point is similar to the criteria used in many experimental cases. One could easily change this limit to $3 H_{c 1}$ or $4 H_{c 1}$ without changing the results, provided that $J_{E}$ is large enough. This can be easily understood from the flat tails in the full hysteresis loop shown in Fig. 2.

The EB field $H_{E B}$, the coercivity $\Delta H$, and the magnetization shift $m_{B}$ are defined as

$$
\begin{gathered}
H_{E B}=\frac{H_{c 1}+H_{c 2}}{2}, \\
\Delta H=H_{c 2}-H_{c 1}, \\
m_{B}=\frac{m_{s 1}+m_{s 2}}{2}=\frac{1+m_{s 2}}{2} .
\end{gathered}
$$

According to Eq. (5), the loops shifted to the left on the $H$ axis (as occurs with the loops in the present paper) will have negative exchange bias field. Figures 3 and 4 show the dependence of $\left\langle\left|H_{E B}\right|\right\rangle$ and $\Delta H$ on $J_{E}$ for $\sigma=1$ and different values of $f$ as indicated by the legend. Even for very low values of $f,\left\langle\left|H_{E B}\right|\right\rangle$ increases and saturates for large enough values of $J_{E}$.

In the case of coercivity (Fig. 4) two important results should be emphasized: the increase (almost $40 \%$ in certain cases) in coercivity for intermediate values of $J_{E}$ and the saturation at a constant value (which depends on $f$ ) for large $J_{E}$. Such limiting values at large $J_{E}$, however, are smaller than the coercivity of the system without exchange enhancement.

In order to analyze the dependence of the system properties on the amount of disorder $\sigma$, we choose a value of $J_{E}$ that is large enough so that $\left\langle\left|H_{E B}\right|\right\rangle$ has reached the limiting maximum value (see Fig. 3). Figures 5 and 6 show the behavior of $\left\langle\left|H_{E B}\right|\right\rangle$ and $\langle\Delta H\rangle$ versus $\sigma$ for $J_{E}=20$ and different values of $f$ as indicated by the legend. Unexpectedly, $\left\langle\left|H_{E B}\right|\right\rangle$ shows nonmonotonic behavior with $\sigma$, first decreasing until a minimum is reached, but which increases slowly for large amounts of disorder.

The marked variation of $\left\langle\left|H_{E B}\right|\right\rangle$ and $\langle\Delta H\rangle$ for values of $f$ between 0.015 and 0.025 when $\sigma \rightarrow 0$ is associated with the fact that the ascending part of the hysteresis loop is very

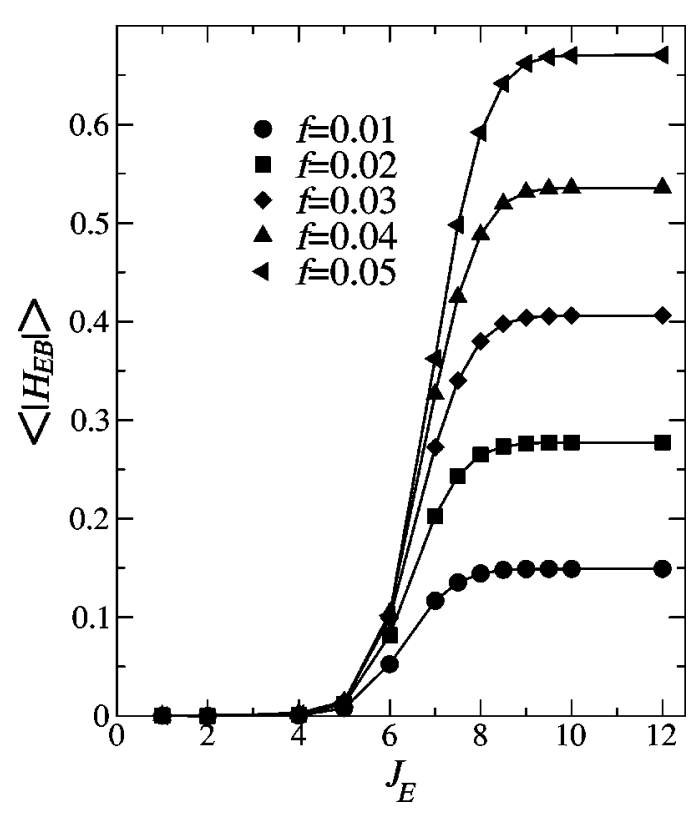

FIG. 3. Dependence of the exchange bias $\left\langle\left|H_{E B}\right|\right\rangle$ on the exchange enhancement $J_{E}$ for $\sigma=1$ and different values of $f$ as indicated by the legend. Data have been obtained by averaging 1000 realizations of a system of size $L=50$. The lines are a guide to the eye. Statistical error bars are smaller than the symbol size.

sensitive to the existence of nuclei of unreversed spins in the negative magnetized state. For very low values of $f$ we expect that all the nuclei of unreversed spins will be formed by two positive spins joined by an enhanced bond. The negative spins surrounding such a nucleus will flip (in the $\sigma \rightarrow 0$ limit) around $H=2$ [see Eq. (4)]. However, for larger values of $f$,

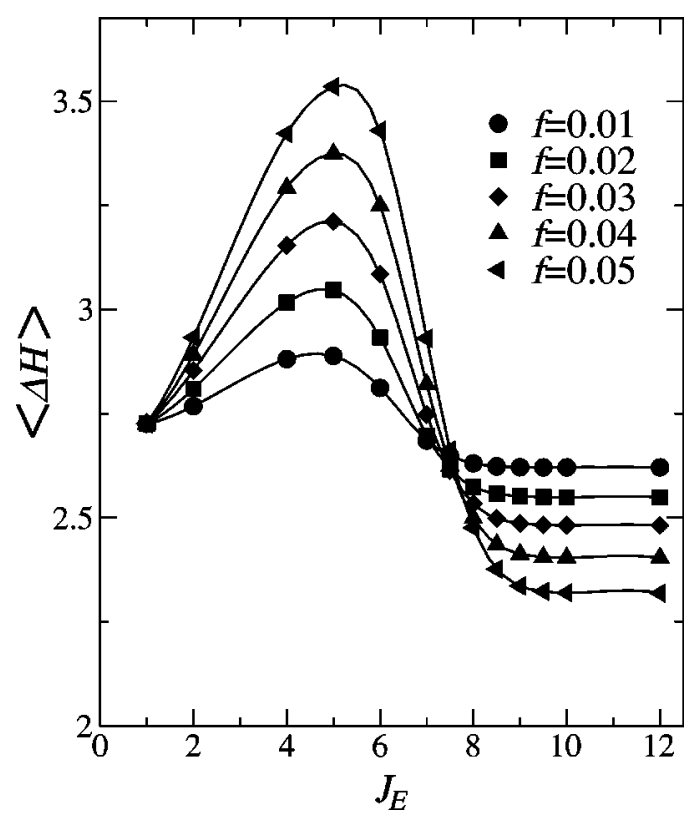

FIG. 4. Dependence of the coercivity $\langle\Delta H\rangle$ on the exchange enhancement $J_{E}$ for $\sigma=1$ and different values of $f$ as indicated by the legend. Data have been obtained by averaging 1000 realizations of a system of size $L=50$. The lines are a guide to the eye. Statistical error bars are smaller than the symbol size. 


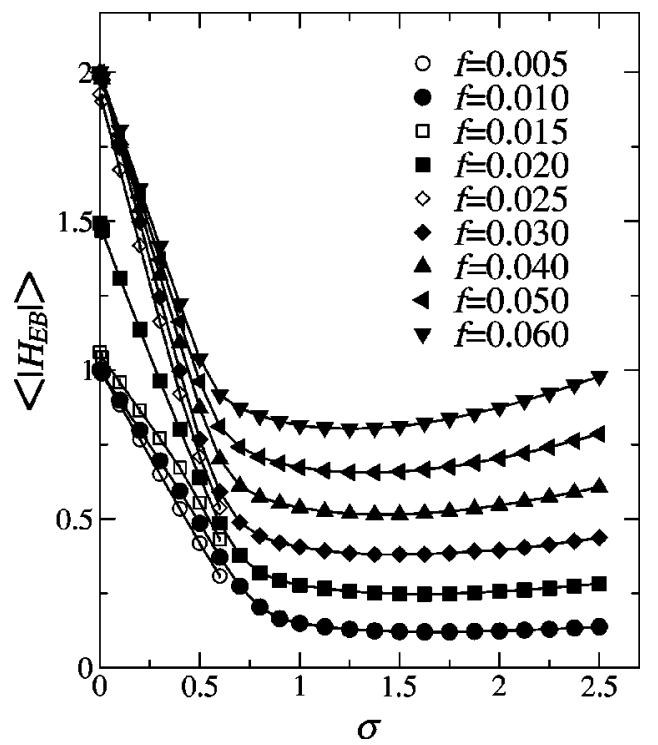

FIG. 5. Dependence of the exchange bias $\left\langle\left|H_{E B}\right|\right\rangle$ on the amount of disorder $\sigma$ for $J_{E}=20$ and different values of $f$ as indicated by the legend. Data have been obtained by averaging 1000 realizations of a system of size $L=50$. The lines are a guide to the eye. Statistical error bars are smaller than the symbol size.

larger nuclei will exist. For instance, a nucleus formed by three spins joined by two perpendicular bonds, both with exchange enhancement, acts as a nucleating seed which triggers the avalanche towards the positive magnetization phase when $H=0$. When $f$ is large enough, such that the probability for such nuclei is significantly different from zero, the coercive field for the ascending branch decreases from 2 to 0 , thus increasing $H_{E B}$ and decreasing $\Delta H$.

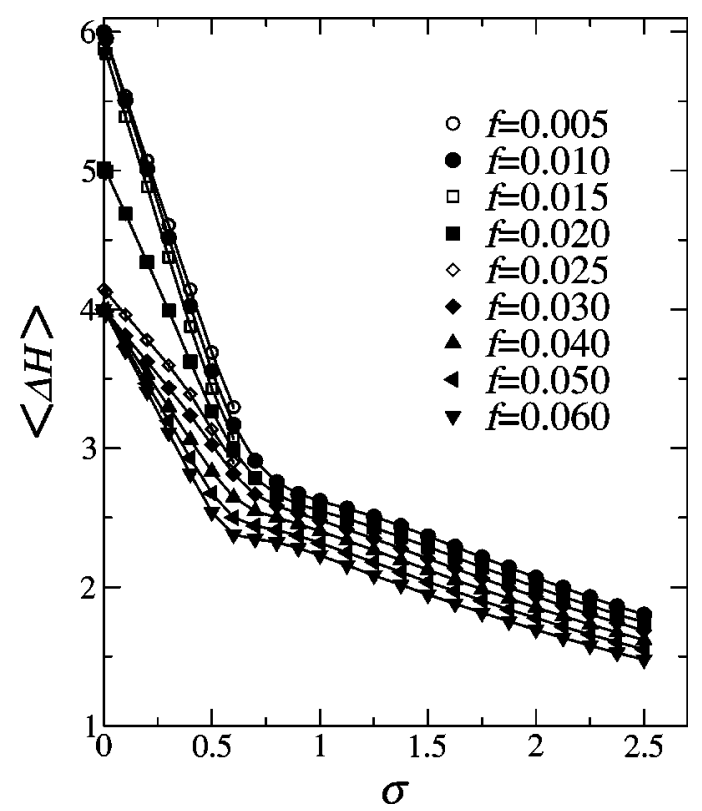

FIG. 6. Dependence of the coercivity $\langle\Delta H\rangle$ on the amount of disorder $\sigma$ for $J_{E}=20$ and different values of $f$ as indicated by the legend. Data have been obtained by averaging 1000 realizations of a system of size $L=50$. The lines are a guide to the eye. Statistical error bars are smaller than the symbol size.

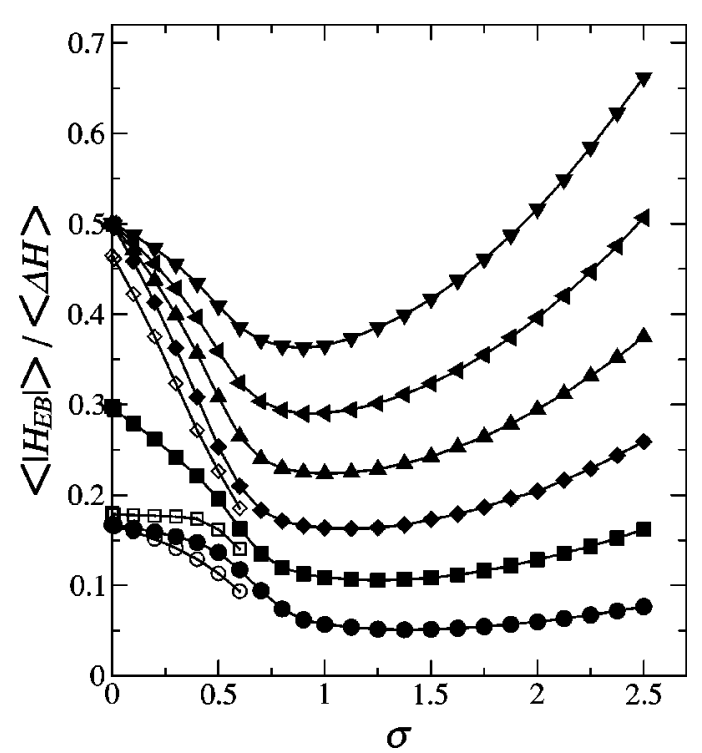

FIG. 7. Dependence of the relative exchange bias $\left\langle\left|H_{E B}\right|\right\rangle /\langle\Delta H\rangle$ on the amount of disorder $\sigma$ for $J_{E}=20$ and different values of $f$ as indicated by the legend in Fig. 6. Data have been obtained by averaging 1000 realizations of a system of size $L$ $=50$. The lines are a guide to the eye. Statistical error bars are smaller than the symbol size.

The nonmonotonic behavior of the EB when disorder is increased is better seen in Fig. 7 by plotting $\left\langle\left|H_{E B}\right|\right\rangle /\langle\Delta H\rangle$, which is a dimensionless quantity and is more relevant from the experimental point of view. Note that for large $f$ and $\sigma$, we can find strongly biased hysteresis loops for which $H_{c 1}$ and $H_{c 2}$ are negative. An example, obtained by sweeping the field between $H= \pm 4.5$, is shown in Fig. 8 .

\section{DISCUSSION}

One of the reasons for the present work was to obtain hysteresis loops with EB by modifying the zero-temperature RFIM as little as possible. The straightforward, naive idea would be to consider nonsymmetric distributions of random fields, for which the average $\left\langle h_{i}\right\rangle \neq 0$ will create the displacement of the loop. From our point of view, this will correspond to the effect of an uncompensated AFM layer. Constraining ourselves to the inclusion of compensated disorder, we have found that any symmetric distribution of random fields cannot give EB. To understand this, suppose that a certain fraction of spins is pinned by very positive and (symmetrically) very negative random fields. Figure 9(a) shows the schematic hysteresis loop corresponding to such a system. The spins with more positive random fields, which are the last to reverse in the decreasing branch, will be the first to flip in the increasing branch of the full hysteresis loop. Therefore, the full hysteresis loop (symmetric, without EB) will overlap with the minor hysteresis loop, as shown in Fig. 9(a). In contrast, when the bond distribution is distorted, as is done in the model presented in this work [and which preserves the symmetry properties of the Hamiltonian in Eq. 


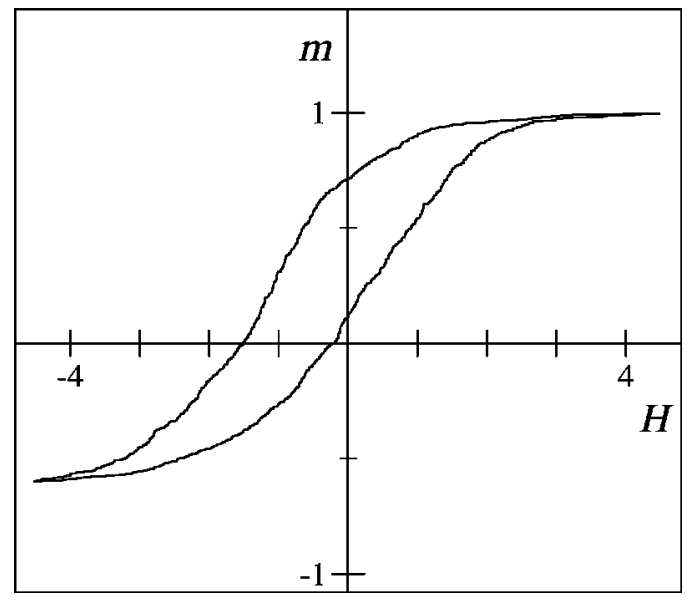

FIG. 8. Hysteresis loop exhibiting exchange bias obtained from a numerical simulation of a $L=50$ system with $J_{E}=20, f=0.05$, and $\sigma=3$. The external field has been cycled between $H= \pm 4.5$.

(1)], the spins with larger ferromagnetic coupling will be the last to reverse in the decreasing branch and also the last to be reversed in the increasing branch. Thus, minor loops will not coincide with the full loops and will easily exhibit large EB as indicated in Fig. 9(b).

The justification of the proposed model would require a physical explanation for the exchange enhancement phenomena. Recently it has been suggested that spin waves in the FM/AFM interface could be responsible for an enhancement of the exchange coupling. ${ }^{22}$ Here we propose a different mechanism based on the existence of quenched disorder in the AFM layer. The most common case is the existence of antiphase domain boundaries as considered in Refs. 9 and 23-25. One can assume that the exchange interaction between the magnetic moments in the FM layer has two contributions, the first coming from the direct overlap of the electronic wave functions of the atoms in the FM layer and a second arising from a superexchange interaction through the overlap with the electrons in the AFM layer. The existence of quenched disorder in the AFM layer can modify this second contribution of the exchange interaction giving rise to the exchange enhancement. Since the energies associated with the broken AFM bonds can be higher than the FM exchange energies (for instance, due to the existence of strong anisotropy), it is plausible to imagine that the defects in the AFM layer can influence the FM exchange interactions.

We would like to give a possible mathematical formulation for such a physical mechanism within the framework of lattice models. Let us consider that the effective exchange interaction between two neighboring spins $S_{i}$ and $S_{j}$ of the FM layer is given by

$$
E_{i j}=-J_{0} S_{i} S_{j}-K S_{i} S_{j} \sigma_{i} \sigma_{j},
$$

where $\sigma_{i}$ and $\sigma_{j}$ are the spin variables describing the magnetic moments in the AFM layer that sit exactly below the $S_{i}$ and $S_{j}$ spins of the FM layer. Notice that other interaction terms such as $S_{i} \sigma_{i}$ are not considered here since they do not modify the effective exchange energy, but contribute to the random-field terms as mentioned in Sec. II. The constant
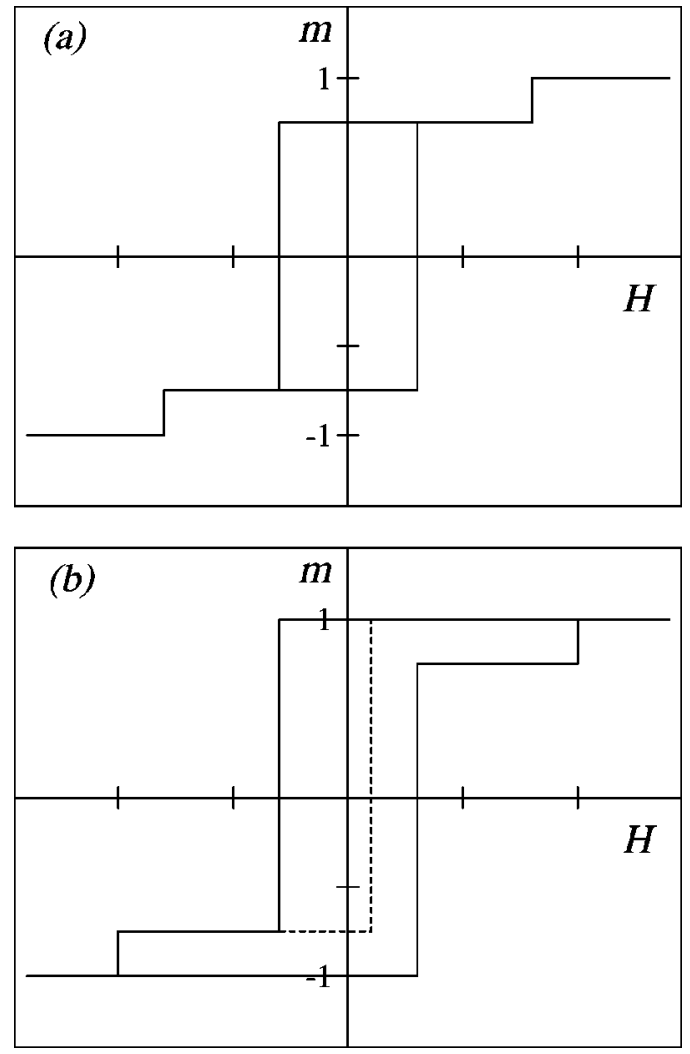

FIG. 9. Schematic examples of the hysteresis loops obtained for a model with strongly enhanced $\pm h$ random fields and for strongly enhanced random bonds. In both cases the full loop is symmetric, but the minor loop only exhibits EB for the enhanced random bonds.

$J_{0}>0$ accounts for the exchange interactions in the free FM layer and $K>0$ accounts for the coupling between the two layers. This coupling term can include, in an effective form, the interactions with many atomic layers in the antiferromagnetism. Figure 10 shows a schematic representation of the spins of the FM and the AFM layers in two situations: Fig. 10 (a) corresponds to the normal situation for an ordered AFM layer, whereas Fig. 10(b) corresponds to the case in which the AFM layer exhibits an antiphase domain boundary.

In Fig. 10(a), the energy of the $i, j$ pair is $E_{i j}=-\left(J_{0}\right.$ $-K) S_{i} S_{j}$, whereas in Fig. 10(b) (or on any antiphase boundary) the energy is given by $E_{i j}=-\left(J_{0}+K\right) S_{i} S_{j}$. The ratio between the two exchange constants is $J_{E}=\left(J_{0}+K\right) /\left(J_{0}\right.$ $-K$ ), which can be much larger than 1 when $K$ is close to but smaller than $J_{0}$. Experimental evidence that the value of $K$ can be of the same order of magnitude as $J_{0}$ are, to our knowledge, not available. This may indicate that Eq. (8), while capturing the correct physics at a qualitative level, could be a too simple description of the complex interactions between the FM and the AFM layers.

\section{COMPARISON WITH EXPERIMENT}

The idea that exchange bias could be a minor loop effect was already suggested to explain the influence of the AFM 


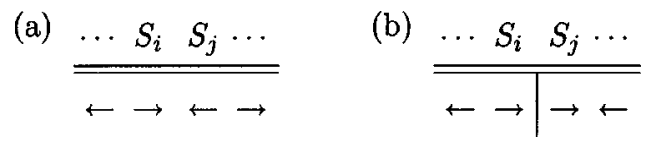

FIG. 10. Schematic representation of two spins $S_{i}$ and $S_{j}$ of the FM layer on (a) an ordered AFM layer and (b) an antiphase domain boundary.

spin flop in EB. ${ }^{26}$ Moreover, it has been shown experimentally that the uncompensated spins, which have been suggested to result in exchange bias ${ }^{27}$ and which could result in the proposed exchange enhancement, can be reversed at high enough fields ${ }^{17}$ (much higher than those usually used in exchange bias studies). Hence, in this case EB could also be considered as a minor loop effect to a certain extent.

Moreover, the peak observed in the behavior of the coercivity with $J_{E}$ (see Fig. 4) could be correlated with the experimental observation of peaks in the coercive field $H_{c}\left(t_{A F M}\right)$ (where $t_{A F M}$ is the thickness of the AFM layer) close to the critical AFM thickness for the onset of EB or in $H_{c}(T)$ close to the Néel temperature (see Figs. 10 and 13 in Ref. 6). One could consider that as the AFM thickness or the temperature increases, the FM-AFM coupling, which causes the enhancement of $J_{E}$, decreases and is consequently the origin of the observed $H_{c}$ increase, in agreement with theory. Similarly, the changes in $\left\langle H_{E B}\right\rangle$ and $\langle\Delta H\rangle$ with $J_{E}$ could also be correlated with the behavior experimentally observed in $\mathrm{Fe} / \mathrm{MnF}_{2}$ under a cooling field. ${ }^{12}$

Another interesting result of this model is the behavior of EB with the amount of disorder $\sigma$, as seen in Figs. 5 and 7 . Experimentally the role played by disorder in EB is not clear. In some cases there is evidence that increasing disorder increases $H_{E B}$, whereas in other cases the opposite effect has been found ${ }^{28}$ Our model is able to explain both possibilities. Moreover, it is noteworthy that experimental nonmonotonic dependence of $H_{E B}$ on disorder (e.g., roughness, irradiation damage, or structural disorder) has also been reported. ${ }^{9,29,30}$

Finally, another remarkable result from our model is that the loops exhibiting EB also exhibit a vertical shift in the magnetization axis. This effect has been observed experimentally. ${ }^{24,28,31-33}$ However, since the fraction $f$ is very small, the shift can also be very small and in some cases, would be difficult to observe experimentally. Figure 11 shows the dependence of the magnetization shift $\left\langle m_{B}\right\rangle$ on $\left\langle\left|H_{E B}\right|\right\rangle$ for different values of $f$. The points correspond to different values of $\sigma$ ranging from 0 to 2 . In view of these results, it would be interesting to measure such displacements in different experimental systems.

\section{SUMMARY AND CONCLUSIONS}

We have presented a simple model for the study of exchange bias. The model is based on the RFIM driven by an

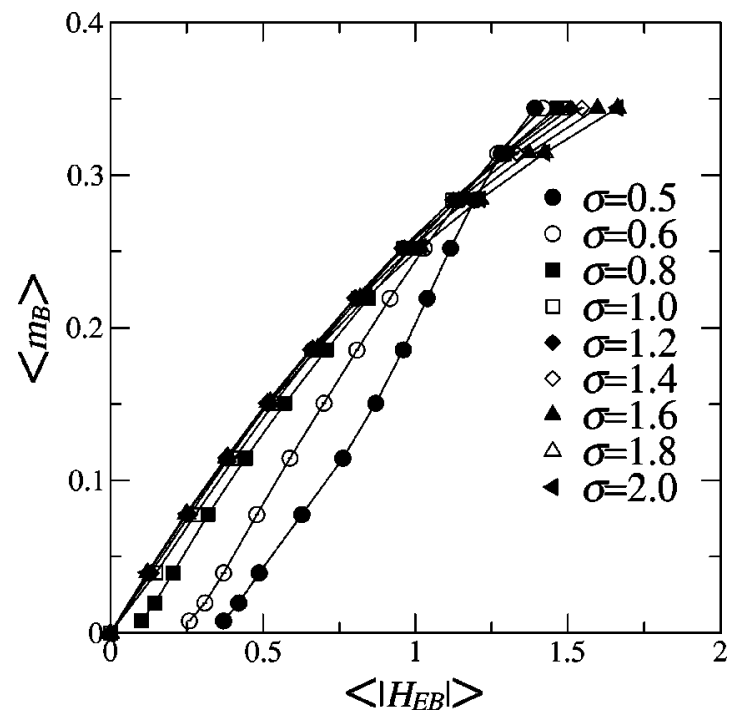

FIG. 11. Magnetization shift $m_{B}$ versus $H_{E B}$ for different values of $f$ as indicated by the legend. The points correspond to systems with different values of $\sigma$, ranging from 0 to 2 . The lines are a guide to the eye. Statistical error bars are smaller than the symbol size.

external field with metastable dynamics. The key ingredient is the existence of a tiny fraction of ferromagnetic bonds, which are strongly enhanced. This creates long reversible plateaux in the decreasing branch. When this pseudosaturated state is reached, the reversal of the field gives rise to an EB loop. Different properties of these loops have been computed as a function of the fraction of enhanced bonds and the amount of disorder in the system. We have suggested a possible physical mechanism to justify the existence of such an exchange enhancement in a FM layer on a AFM layer with antiphase domain boundaries, based on the existence of superexchange coupling between the two layers.

The main conclusions of the paper are (i) EB is due to a minor loop effect, (ii) compensated AFM layers can exhibit exchange biased loops with a concomitant magnetization shift, and (iii) many experimental phenomena related to exchange bias such as peaks in the coercive field, magnetization shifts, a marked coercivity increase, or nonmonotonic dependence of $H_{E B}$ on disorder, can successfully be reproduced with this model.

\section{ACKNOWLEDGMENTS}

We acknowledge many fruitful discussions with $\mathrm{J}$. Nogués. This work has received financial support from CICyT (Spain), Project No. MAT2001-3251 and CIRIT (Catalonia), Project No. 2000SGR00025. X.I acknowledges financial support from DGICyT.
*Electronic address: xit@ecm.ub.es
†Electronic address: eduard@ecm.ub.es
${ }^{\dagger}$ Electronic address: toni @ecm.ub.es
${ }^{1}$ G. Bertotti, Hysteresis in Magnetism, Electromagnetism series
(Academic, San Diego, 1998).

${ }^{2}$ J.P. Sethna, K. Dahmen, S. Kartha, J.A. Krumhansl, B.W. Roberts, and J.D. Shore, Phys. Rev. Lett. 70, 3347 (1993).

${ }^{3}$ E. Vives and A. Planes, Phys. Rev. B 50, 3839 (1994). 
${ }^{4}$ E. Obradó, E. Vives, and A. Planes, Phys. Rev. B 59, 13901 (1999).

${ }^{5}$ E. Vives and A. Planes, Phys. Rev. B 63, 134431 (2001).

${ }^{6}$ J. Nogués and I.K. Schuller, J. Magn. Magn. Mater. 192, 203 (1999)

${ }^{7}$ A.E. Berkowitz and K. Takano, J. Magn. Magn. Mater. 200, 552 (1999).

${ }^{8}$ B. Dieny, V.S. Speriosu, S.S.P. Parkin, B.A. Gurney, D.R. Wilhoit, and D. Mauri, Phys. Rev. B 43, 1297 (1991).

${ }^{9}$ P. Miltényi, M. Gierlings, J. Keller, B. Beschoten, G. Güntherodt, U. Nowak, and K.D. Usadel, Phys. Rev. Lett. 84, 4224 (2000).

${ }^{10}$ G. Li, T. Yang, Q. Hu, and W. Lai, Appl. Phys. Lett. 77, 1032 (2000).

${ }^{11}$ J. Nogués, D. Lederman, T.J. Moran, and I.K. Schuller, Phys. Rev. Lett. 76, 4624 (1996).

${ }^{12}$ C. Leighton, J. Nogués, B.J. Jönson-Åkerman, and I.K. Schuller, Phys. Rev. Lett. 84, 3466 (2000).

${ }^{13}$ S. Gangopadhyay, G.C. Hadjipanayis, C.M. Sorensen, and K.J. Klabunde, J. Appl. Phys. 73, 6964 (1993).

${ }^{14}$ W.H. Meiklejohn, J. Appl. Phys. 33, 1328 (1962).

${ }^{15}$ M. Kiwi, J. Magn. Magn. Mater. 234, 584 (2001).

${ }^{16}$ R.L. Stamps, J. Phys. D 33, R247 (2000).

${ }^{17}$ T. Ambrose and C.L. Chien, Phys. Rev. Lett. 76, 1743 (1996).

${ }^{18}$ A.P. Malozemoff, Phys. Rev. B 35, 3679 (1987).

${ }^{19}$ D. Mauri, H.C. Siegmann, P.S. Bagus, and E. Kay, J. Appl. Phys. 62, 3047 (1987).
${ }^{20}$ M. Kiwi, J. Mejía-López, R.D. Portugal, and R. Ramírez, Europhys. Lett. 48, 573 (1999).

${ }^{21}$ N.C. Koon, Phys. Rev. Lett. 78, 4865 (1997).

${ }^{22}$ H. Suhl and I.K. Schuller, Phys. Rev. B 58, 258 (1998).

${ }^{23}$ U. Nowak, K.D. Usadel, J. Keller, P. Miltényi, B. Beschoten, and G. Güntherodt, Phys. Rev. B 66, 014430 (2002).

${ }^{24}$ J. Keller, P. Miltényi, B. Beschoten, G. Güntherodt, U. Nowak, and K.D. Usadel, Phys. Rev. B 66, 014431 (2002).

${ }^{25}$ U. Nowak, A. Misra, and K.D. Usadel, J. Magn. Magn. Mater. 240, 243 (2002).

${ }^{26}$ J. Nogués, L. Morellón, C. Leighton, M.R. Ibarra, and I.K. Schuller, Phys. Rev. B 61, R6455 (2000).

${ }^{27}$ K. Takano, R.H. Kodama, A.E. Berkowitz, W. Cao, and G. Thomas, Phys. Rev. Lett. 79, 1130 (1997).

${ }^{28}$ J. Nogués, T.J. Moran, D. Lederman, I.K. Schuller, and K.V. Rao, Phys. Rev. B 59, 6984 (1999).

${ }^{29}$ C. Leighton, J. Nogués, H. Suhl, and I.K. Schuller, Phys. Rev. B 60, 12837 (1999).

${ }^{30}$ A. Mougin, T. Mewes, R. Lopusnik, M. Jung, D. Engel, A. Ehresmann, H. Schmoranzer, and B. Hillebrads, IEEE Trans. Magn. 36, 2647 (2000).

${ }^{31}$ W.H. Meiklejohn and C.P. Bean, Phys. Rev. 105, 904 (1957).

${ }^{32}$ F. Radu, M. Etzkorn, R. Siebrecht, T. Schmitte, K. Westerholt, and H. Zabel, cond-mat/0204607 (unpublished).

${ }^{33}$ J. Nogués, C. Leighton, and I.K. Schuller, Phys. Rev. B 61, 1315 (2000). 